\title{
Pattern Visualization Through Detection Plane Generation for Macroscopic Imagery
}

\author{
Hanan Hassan Ali Adlan ${ }^{1,2}$ \\ Dept. of Computer Science ${ }^{1,2}$, \\ Faculty of Mathematical Science, U.of K. \\ Khartoum, Sudan ${ }^{1}$ \\ Faculty of Computer and Information Science, PNU \\ Riyadh, Saudi Arabia ${ }^{2}$
}

\begin{abstract}
Macroscopic images are kind of environments in which complex patterns are present. Satellite images are one of these classes where many patterns are present. This fact reflects the challenges in detecting patterns present in this kind of environments. SPOT1b satellite images provide valuable information. These images are affordable and can be applicable in wide applications. This paper demonstrates an approach to generate detection plane that visualize patterns present in the satellite image. The detection plane uses rough neural network to provide optimal representation in backpropagation architecture. Rough set theory combined with multilayer perceptron constitutes the rough neural network. Reduction in the feature dimensionality via the rough module improves the recognition ability of the neural network. It is found that the rough module provides the neural network with optimal features. The ability of the neural network to efficiently detect and visualize the pattern stems from a developed extraction algorithm. The result of the hybrid architecture provides the plane with the best features that visualize the phenomena under investigation. Together with the novel extraction algorithm, the developed system provides a tool to visualize patterns present in SPOT1b Satellite image.
\end{abstract}

Keywords-pattern detection; hybrid architecture; backpropagation networks; rough set; image patterns

\section{INTRODUCTION}

Hybrid architectures are novel techniques to intelligent and powerful systems. The architectures usually present combination of different techniques. Recently rough set theory in combination with neural networks enriches the literature with architectures that enhances the recognition ability of neural networks. Hybrid architectures are formed to overcome limitations of individual system. The power in such systems stem from their capabilities to exhibit multiple information processing $[1,2,3,12]$

Recent developments in the area of image recognition involve methods for extraction, classification, and selections $[4,13,14]$. An open problem in this area is to find the best features that enable success recognition in classification processes.

The best set that can represent features is generally goal, data, and classification design dependent. Complex application problems such as in remote sensing, medical imaging ,...etc are likely to present large numbers of features so a method for reduction is highly desirable $[5,11,14,15]$.
This paper demonstrates an approach to automatic detection plane generation for certain patterns present in SPOT1b satellite image. The paper also provides an automatic feature extraction pseudo code that improves the extraction process in such big data environment. A highlight on the architecture used is provided. The approach is theoretically robust, and found efficient to such complicated image.

\section{THE RNN ARCHITECTURE}

RNN composed of two phases. A feature extraction phase (FE), and a feedforeward neural network phase. The FE accepts an input signal which is two dimensional input image, generates frames for feature computations. The rough module within the FE phase filtered the features. Outputs of the FE are the inputs to the second phase. Neurons in the input layer accept distilled features from the FE phase.

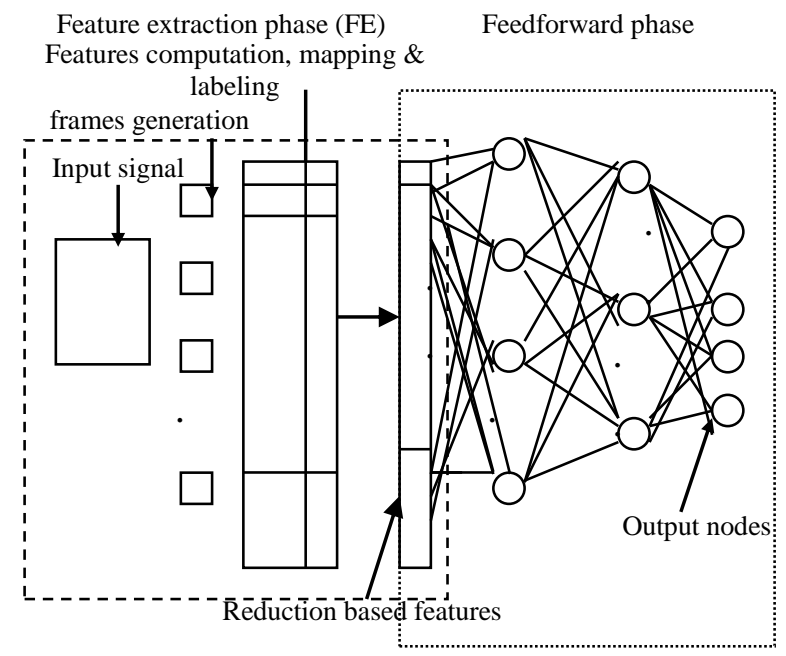

Fig. 1. Rough Neural Network Architecture

Two sets are to be composed from the image, one for training and the other for testing. The network is a backpropagation network. Figure 1 gives configuration of the architecture. [11]

\section{ATOMATIC FEATURE EXTRACTION}

Features extracted from the satellite image assigned labels that distinguish them. For efficient extraction a pseudo code to 
automatically assign the labels to the extracted features has been developed. The extraction process forms frames from the satellite image, and subsequently extract features from these frames. Association of the frame label is done by a 3x3 filter. Whenever the frame matched a filter kernel, a label is created to identify the frame.

The following pseudo code (pseudo code I) illustrates automatic extraction and labeling

Pseudo code I: Automatic Extraction and Labeling

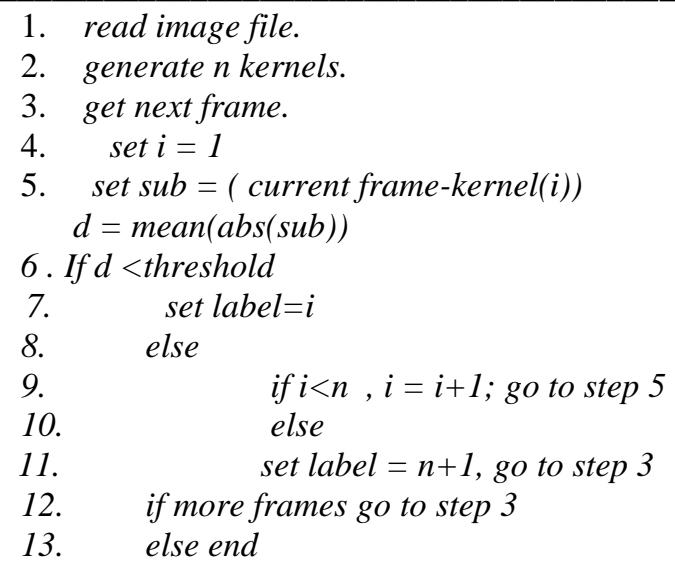

\section{DETECTION PLANE GENERATION}

The detection plane is the plane produced by the network as a result of the present of a pattern in the satellite image. It is considered visualization for the detected phenomena, object,...etc. In this work, we span the image for three categories. The first are water bodies, the second is buildings and networks, and the third is vegetation and swaps. The trained architecture is used in the plane generation. RNN was trained and tested over a portion of $512 \times 512$ from an original satellite image (Figure 2). The original image is $2997 \times 4139$ scene over the area of Sepang, Kajang, Bangi in Malaysia.

In order to creat the detection plane, a source image is supplied to the RNN. Starting at the top left corner, the trained RNN scanned the image horizontally $3 \times 3$ frame at a time. Then it is moved down three rows and moved horizontally again. The process continued until the network traverse the entire image. A 3x3 destination frame is created each time the network moves to a new position. At any position a distance measure is formed from the original frame vector to the network vector, then threshold. The result of the threshold yields the detection plane frame corresponding to the current position. The following pseudo code (Pseudo code II) illustrates the process.

Pseudo code II: Detection plane generation

\section{1 .load net}

2. read image

3. $\quad$ generate detection plane

4. scan image from upper left

5. $\quad$ set $f=$ frame;
6. $\quad f v=\operatorname{extract}(f)$;

7. $\quad \operatorname{red}=\operatorname{reduct}(f v)$;

8. $c=\operatorname{sim}($ net, red $)$;

9. feature detected?

10.

12.

$$
\begin{aligned}
& \text { yes; set corresponding detection } \\
& \text { plane frame }=0
\end{aligned}
$$

\section{plane frame $=1$ \\ Experiments}

The approach described in the previous section illustrated with the SPOT1b satellite image shown in Fig 2. The image is portion of the SPOT1b of the area of Sepang, Bangi, Kajang, in Malayisa. The objective is to recognize three patterns present in the image. The image is $512 \times 512$ pixels of surface from the original scene of $2997 \times 4139$ pixels.

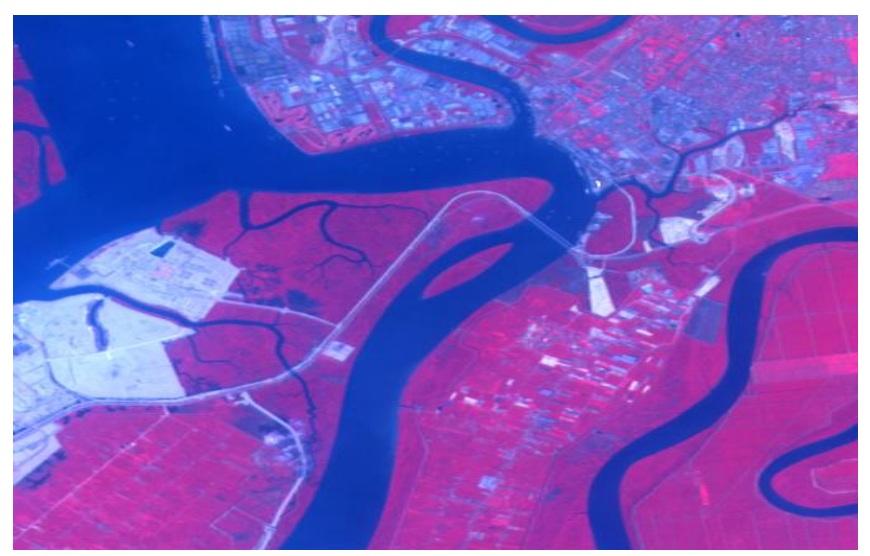

Fig. 2. Portion of spot1b $2997 \times 4139$ scene over the area of Sepang, Kajang, Bangi (Malaysia). The image is $512 \times 512$ pixels

The RNN trained and tested using the 512x512 image. Features used in the recognition system isolated from measures of intensities and intensity variations of the pixels, texture features based on spatial gray level dependence matrices, and moment's invariants. More discussion on this can be found in [10].

Labeling is performed through the mean, standard deviation, and the color. These statistics are computed for each band of the image; in addition to the color, which plays a major factor. Experiments are carried extensively to find the appropriate frame size that best fit in this application. Starting from $16 \times 16,8 \times 8,5 \times 5$, and ends with $3 \times 3$ which is found to be the best frame size. The $5 \times 5$ frame produce very low recognition rate, the best of is $35 \%$. The others fail to represent any of the features. This can be attributed to the pixel resolution of the SPOT sensor. 24 feature detectors representation of the satellite image patterns were extracted from the $3 \times 3$ frames composed from the original scene.

\section{DISSCUSSION}

The architecture is examined for the recognition of the three categories water bodies, buildings and networks, and vegetations. 24 feature detectors representation of the satellite image patterns were extracted from $3 \times 3$ frames composed from the original scene. 
The rough module generated the information system based on the distilled features. The best algorithm suited for this application is found to be the recursive minimal entropy algorithm [9]. 8900 frames were extracted from the image. 5000 of them were used to form the training set. The whole image is used for testing.

Reduction based on Johnson's reducer is given in Table 1. The table displays nine reducts that are considered the filtered features for the recognition process.

TABLE I. JOHNSON REDUCER

\begin{tabular}{|l|l|l|l|}
\hline & Reduct & Support & Length \\
\hline 1 & $\begin{array}{l}\left\{\mathrm{dv}, \mathrm{fme}, \mathrm{mom} 0^{\circ}, \mathrm{entr} 45^{\circ}, \mathrm{con} 0^{\circ}, \mathrm{con}\right. \\
\left.45^{\circ}, \mathrm{con} 0^{\circ}, \mathrm{con} 90^{\circ}, \mathrm{cr} 135^{\circ}\right\}\end{array}$ & 100 & 9 \\
\hline
\end{tabular}

Different architectures are experimented. Hidden neurons of $200,150,100,50,10$ are examined together with varying learning rates of $0.8,0.5,0.1,0.05,0.07,0.01$.

The best architecture found composed of 9 input neurons, resulted from the Johnson algorithm. Neurons in the hidden units are 10, and 4 output neurons. The fourth output neuron accommodates the mixed frames, which the system fails to classify as any of the three categories. This can be attributed to the present of more than one pattern or may represent different pattern. The best architecture's parameters are given in Table 2.

TABLE II. NETWORK PARAMETERS FOR THE BEST ARCHITECTURE IN THE ROUGH NEURAL NETWORKS

\begin{tabular}{|l|lr|}
\hline Network & Parameters & \\
\hline & Performance & \\
& Goal: & 0.01 \\
Hybrid rough backpropagation & Learning rate: & 0.5 \\
network & Epochs: & 15904 \\
& Momentum: & 0.95 \\
& & \\
\hline
\end{tabular}

Table 3 displays the best recognition rates obtained.

TABLE III. ROUGH NEURAL NETWORKS RECOGNITION RATES

\begin{tabular}{|l|l|}
\hline Network & Recognition Rates \\
\hline Training & $98.55 \%$ \\
Generalization & $96.615 \%$ \\
\hline
\end{tabular}

Previous work [11], experimented a backpropagation network of the same parameters, excluding the rough module resulted in $89.076 \%$ recognition rate.

\section{1) Network Scannin}

Network scanning mode is the process of generating the detection plane. The scanning mode results in a visualization plane for certain patterns in the image.

The network scanning mode results in Figures 3 to 5. The figures visualize the three patterns under investigation, and found to be present in the satellite image.

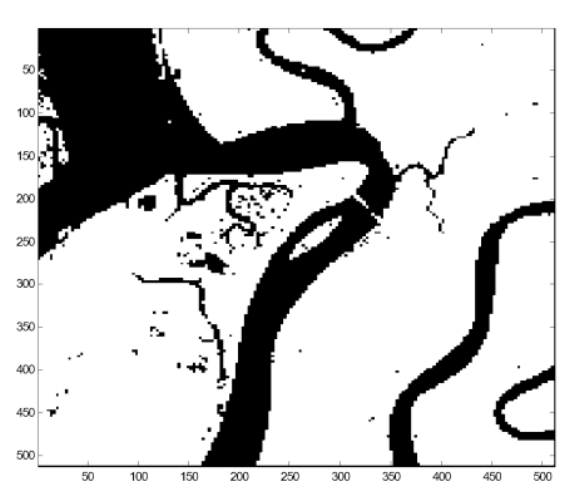

Fig. 3. Detection Plane Representation for the Water bodies

Figure 3 gives visualization of the water bodies. The figure is a result of traversing the image with the neural network in a scan mode. The water bodies appear in black color.

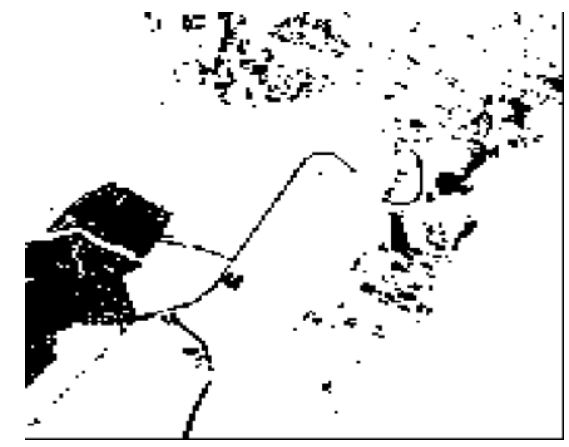

Fig. 4. Detection plane representation for the buildings and networks

The buildings and networks are shown in Figure 4 in black.

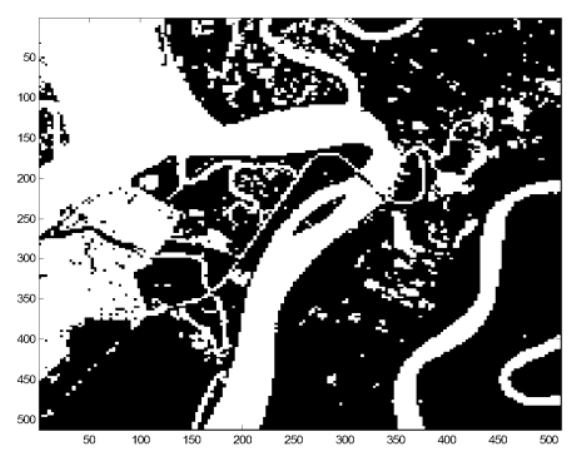

Fig. 5. Detection plane representation for the vegetation and swamps

Figure 5 detect the vegetation and swamps present in the image. The feature appears in black.

\section{CONCLUSIONS}

The work in this paper represents visualization of patterns present in SPOT1b satellite image. Each pattern is visualized in a separate plane.RNN which is found to generalize and deal with uncertainty and vagueness present in the SPOT1bsatellite image is developed to produce a system capable of visualizing the patterns present in the satellite image. 


\section{REFERENCES}

[1] LingasPawan 1998 "Comparison of Neofuzzy and rough neural networks” Information Sciences 110, 207-215.

[2] Yasdi R. 1995 "Combining Rough Sets Learning and Neural Learningmethod to deal with uncertain and imprecise information", Neurocomputing $7,61-84$.

[3] Ruppert George S. and Mathias Schardt 1997, 'A Hybrid Classifier for Remote Sensing Applications'. International Journal of Neural Systems, Vol. 8, No. 1, 63-68.

[4] Hussain B. and Kabuka M. R. 1994 "A Novel Feature Recognition Neural Network and its Application to Character Recognition", IEEE Transactions on Pattern Analysis and Machine Intelligence, Vol. 16, No.1.

[5] Swiniarski Roman W., Hargis Larry, 2001 "Rough sets as a front end of neural-networks texture classifiers". Neurocomputing 36, 85-102.

[6] Khoo Li-Phing, ZhaiLian-Yin, 2001 "A prototype genetic algorithmenhanced rough set-based rule induction system". Computers in industry 46, 95-106.

[7] Swiniarski Roman, 1992. "Intelligent Decision Support, Hand book of applications and advances of the Rough Sets Theory". Kluwer, Netherlands.

[8] Gonzalez Rafael C. and Woods Richard E.,1992, "Digital Image Processing". Addison-Wesley.
[9] Vinterbo S. and Øhrn A. 2000 "Minimal Approximate Hitting Sets and Rule Templates"., International Journal of Approximate Reasoning, 25(2), 124- 143. Elsevier.

[10] Hanan H. A. Adlan, AbdRahmanRamli, AdznanJantan, and BachokTaib. 2003, "Backpropagation for Recognition of Invariants and Spatial Detectors of Constituents of Perceptual Patterns". Brunei Darussalam Journal of Technology and Commerece, Vol. 3, No. 1.

[11] Hanan H. A. Adlan, AbdRahmanRamli, Elsadig Ahmed MohdBabiker. 2005, "Improving Generalization in Backpropagation Networks Architectures". CISAR, International Advanced Technology Congress, Malaysia.

[12] Singh K., Raghuwanshi M. 2009,"Approach to Enhance Performance of Face Recognition Systems Using Rough Sets", in V. Sn_a_sel (Ed.): Digital Technology Journal 2009, Vol. 2, pp. 23\{29, ISSN $\overline{1} 802-5811$ (print), ISSN 1802-582X (online).

[13] Gupta S. and Patnaik K. 2008, "Enhancing Performance of Face Recognition System by Using Near Set Approach for Selecting Facial Features", Journal of Theoretical and Applied Information Technology, (C) 2005 - 2008 JATIT. All rights reserved.

[14] Meng Yang, Lei Zhang, Simon Chi-Keung Shiu, and David Zhang, 2013. "Robust Kernel Representation with Statistical Local Features for Face recognition", IEEE Transactions on Neural Networks and Learning Systems, vol. 24, No. 6 June

[15] SamanRazavi and Bryan A. Tolson, 2011. " A New Formulation for Feedforward Neural Networks", IEEE Transactions on Neural Networks vol. 22 No. 10, October 2011. 